\title{
Numerical modelling of the hydrodynamic ram phenomenon
}

\author{
D. Varas, R. Zaera, J. López-Puente* \\ Department of Continuum Mechanics and Structural Analysis, University Carlos III of Madrid, Avda. de la Universidad, 30, 28911 Leganés, Madrid, Spain
}

\begin{abstract}
A B S T R A C T
Hydrodynamic ram (HRAM) is a phenomenon that occurs when a high kinetic energy object penetrates a fluid filled container. The projectile transfers its momentum and kinetic energy through the fluid to the surrounding structure, increasing the risk of catastrophic failure and excessive structural damage. This is of particular concern in the design of wing fuel tanks for aircraft since it has been identified as one of the important factors in aircraft vulnerability. In the present paper, the commercial finite element code LS DYNA has been used to simulate an HRAM event created by a steel spherical projectile impacting a water filled aluminium square tube. Two different formulations (ALE and SPH) are employed to reproduce the event. Experimental tests which indicate the pressure at different points of the fluid, displacement of the walls and cavity evolution for different impact velocities are compared with the numerical results in order to assess the validity and accuracy of both ALE and SPH techniques in reproducing such a complex phenomenon.
\end{abstract}

\section{Introduction}

The process by which a high speed projectile penetrates a fluid filled tank and transfers kinetic energy to the surrounding walls is known as hydrodynamic ram (HRAM). The HRAM effect in fuel tanks is identified as one of the important factors in aircraft vulnerability since the fuel tanks represent the largest exposed area of all the vulnerable components. HRAM is especially dangerous for aircraft with extremely lightweight designs. These commonly use wing integral fuel tanks, in which structural resistance cannot be improved by strengthening the airframe since this would coun teract the requirements of a lightweight design.

Vulnerability to HRAM is usually, but not exclusively, related to military aircraft. In 1990 the Federal Aviation Administration (FAA) established the Aircraft Catastrophic Failure Prevention Research Program. One research area of this program was the analysis of the effects of an uncontained turbine engine fragment penetrating aircraft fuel tanks [1]. In 2000, when a Concorde crashed after takeoff from Charles de Gaulle Airport (France), the final investi gation report revealed that the HRAM had played a significant role in the aircraft failure.

Hydrodynamic ram consists of four principal stages: shock, drag, cavitation and exit (Fig. 1). Each stage contributes to structural damage in a different way and to a different extent. When the projectile penetrates the wall of the fluid filled structure, the impact energy is transferred to the fluid, generating a high

\footnotetext{
* Corresponding author. Fax: +34 916249430.

E-mail address: jlpuente@ing.uc3m.es (J. López-Puente).
}

pressure hemispherical shock wave. This leads to damage primarily in the vicinity of the impact position. During the drag phase, the projectile travels through the fluid, while its kinetic energy is partially transformed into fluid motion as the projectile is slowed by fluid drag forces. The displacement of the fluid from the projectile path generates a radial pressure field. In contrast to the pressure field that develops during the shock phase, the fluid is accelerated gradually instead of impulsively. This causes less intense peak pressures but of greater temporal extent. The displacement of fluid during the drag stage forms a cavity behind the projectile. The subsequent expansion and collapse (oscillations) of the cavity are known as the cavitation stage. The oscillations of the cavity can cause significant pressure pulses. The final stage occurs when the projectile exits the container. In contrast to the perforation of the front wall, the exit of the projectile occurs through a pre stressed wall, caused by the initial shock stage and the subsequent loading by the fluid.

Simulation of HRAM events has been attempted for over 30 years. The first methods employed to simulate HRAM were based on the use of the Piston Theory for the fluid structure interaction. This theory assumes the normal reflection of pressure waves when reaching the walls of the structure, resulting in a one dimensional response mechanism. Ball [2,3] incorporated the Piston Theory into two structural analysis codes, BR 1 and SATANS (Static And Tran sient Analysis, Nonlinear, Shells), in order to simulate the HRAM phenomenon. Numerical results provided by these two codes were compared with experimental data [4 6]. It was found that experi mental strain data and wall deflections greatly exceeded the numerical values. The disagreement was attributed to the failure of the Piston Theory for hydrodynamic ram pressure loading. 


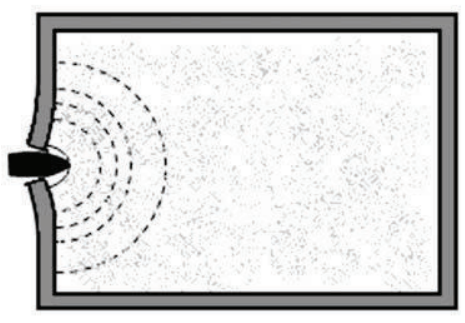

SHOCK

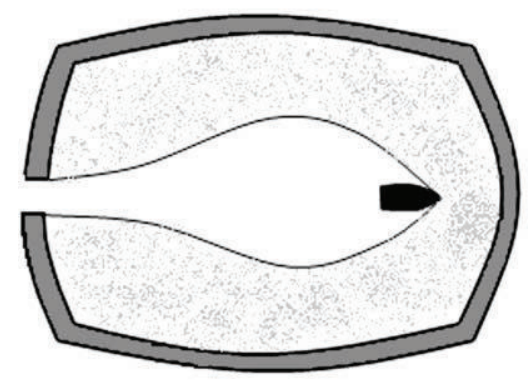

CAVITATION

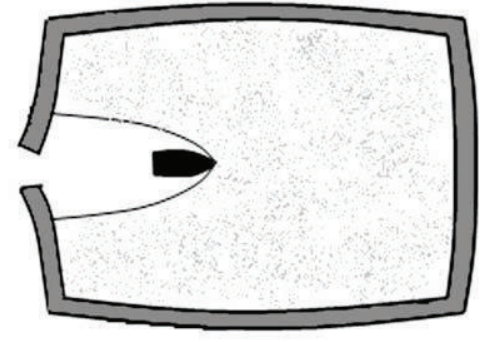

DRAG

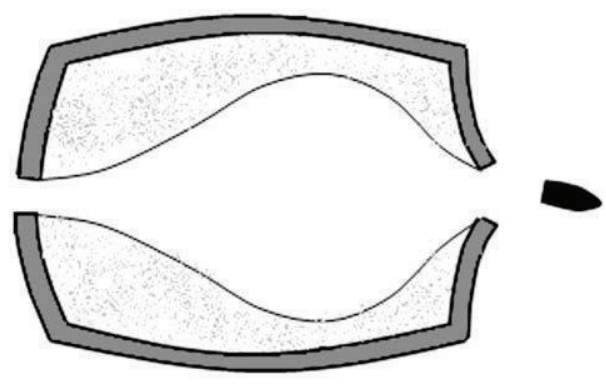

EXIT

Fig. 1. Phases of hydrodynamic ram.

Once it was proved that the Piston Theory failed to predict HRAM fluid structure interaction, Lundstrom [7] proposed the Variable Image Method. Lundstrom described the flow field in terms of a potential function $\phi$ which satisfied the wave equation. The model attempts to approximate the effect of the projectile and cavity by a line of sources distributed along the trajectory behind the projectile. This allows determining the incident pressure wave magnitude by means of Bernoulli's equation and then uses it to calculate the pressure wave reflected from the structures' walls, considering $\phi_{\mathrm{r}} \quad Q \widetilde{\phi}_{i}$; where $\phi_{\mathrm{r}}$ is the potential of the reflected wave, $Q$ is a function that varies in space and time such that $\phi_{\mathrm{r}}$ satisfies the wave equation and $\widetilde{\phi}_{i}$ is the mirror image of the potential of the incident wave about the plane of the wall. $Q$ is determined by matching the fluid velocity with the wall velocity at the interface [8]. In general, this methodology has not been particularly successful and the geometries that can be analyzed are limited. The method is based on the potential functions and is essentially limited to two dimensions. However, the Variable Image Method solved some of the planar assumptions in the Piston Theory model, and provided a more realistic coupling between the fluid and the structure. Other researchers [9] developed a plastic deformation model to predict the structural response of fuel cell walls due to HRAM in contrast to other works in which only the elastic regime was considered. The model requires the knowledge of the total impulse imparted by the fluid to the fuel cell.

Other codes such as HRSR (Hydraulic Ram Structural Response) [10], ERAM or EHRSR were developed [11], but all of them showed their limitations and lack of accuracy on predicting the conse quences of an HRAM event since none of them fully coupled the mechanisms of fluid structure interaction, nor did they allow for a complex, engineered structure.

One of the first documented examples of an HRAM simulation using the full set of continuum equations (e.g., conservation of mass, momentum and energy) and material constitutive descrip tions was performed by Kimsey [12]. In that paper, Kimsey applied a Lagrangian finite element method (the EPIC 2 code) to simulate the penetration of a steel rod into a cylindrical tank. For large physical deformations, the elements may become too distorted, compromising the numerical accuracy of the simulation and the stable time step size, increasing the CPU time. Distortions were a problem in Kimsey's simulation; nevertheless, qualitatively good results were obtained. The main reason for the limitation of Lagrangian codes to be applied in a full HRAM problem is the distortion of the mesh. Eulerian codes, which have a fixed grid system, have no distortion problem. However, the complicated physics and mechanics of HRAM phenomena were not satisfactorily solved until higher order numerical algorithms were incorporated into the codes in the late 1980s.

The Coupled Euler Lagrange methods have been under devel opment since the early to mid 1990s. They are a new generation of computer codes which combine the desirable characteristics of Lagrangian and Eulerian formulations. These methods are being used in simulations where fluids interact with structures or when high distortions may appear [1,13 17]. The Arbitrary Lagrangian Eulerian (ALE) technique can be viewed as a hybrid between the Lagrangian and Eulerian methods. When applied to a fluid struc ture interaction problem such as HRAM, the ALE method begins working as a Lagrangian finite element code in which the motions of the fluid and the structure are determined and the finite element grid is deformed following the material. In the ALE methodology, however, the distorted mesh can be partially restored to its original shape based on predefined criteria for element deformation. Following a prescribed measure of permissible distortion in the element, the element shape is changed; and mass, momentum and energy are fluxed across the old element boundaries to calculate their new values for the new element shape. In general, the ALE methodology permits flow of material across element boundaries preserving a balance for the physical velocity between grid motion and flow [11].

Another technique developed for calculation of fluid flow and large deformation in structures is Smoothed Particle Hydrody namics (SPH). At the beginning, the basic SPH method was created by Lucy [20] and Gingold and Monaghan [21] in order to study fission in rotating stars. Later fluid flow applications appeared as authors examined its use in modelling Newtonian, Eulerian and Navier Stokes equations in fluid flow problems. In the SPH method, the conservation equations are applied to discrete particles in the computational domain. The particles are not associated with 
a traditional grid system, therefore, they are not fixed by connec tivity constraints as happens in finite elements. This fact gives them the possibility to freely move and deform in any manner, making SPH especially suitable to simulate processes in which large deformations appear. Application of SPH requires specifying a weight function that describes a domain of influence in which any particle can find the surrounding particles. When applied in this way, SPH has similarities with a free Lagrangian finite element method, where nodes of the grid correspond to the SPH particles. Even though a computational expense to search and locate nearest neighbour particles within the domain of influence is added, SPH combined with a Lagrangian method provides an alternative to the ALE approach so that small distortions are computed using the Lagrangian method while the large distortions are handled with the SPH technique. Examples of the use of this approach to simulate fluid structure or HRAM problems are found in the literature $[13,19,22,23]$. Although the application of SPH in fluid structure problems is relatively recent, this method has been applied to problems in which large deformations occur, as hyper velocity impacts, since some time ago [24 28].

The modelling of coupled problems of fluid structure interac tion such as HRAM has been proven to be a complicated task and is still quite challenging. The suitability and predictive capabilities of the mentioned techniques (ALE and SPH) in an HRAM problem have not yet been solved. This is of great importance since analytical solutions can provide only a limited understanding of the nature of the behaviour. ALE and SPH techniques have been compared to other approaches and even between them, although not always in fluid structure problems. Sometimes, they have been compared without any experimental data, and other times, the available data is not as complete as would be desired.

In the present paper, numerical simulations of a water filled aluminium square tube subjected to impact by steel spherical projectiles at different velocities are shown. The simulations are performed with the software LS DYNA using two different tech niques for the fluid phase: the ALE formulation and the SPH method. Experimental tests providing the pressure in different points of the fluid, deformation of the walls and cavity evolution for different impact velocities are compared with the numerical results in order to assess the validity and accuracy of both ALE and SPH techniques in reproducing such a complex phenomenon. The analysis of the results will reveal the most appropriate technique to simulate HRAM.

\section{Experimental setup}

To achieve an appropriate and wide validation of the numerical modelling, it is necessary to have enough experimental data. As there is not much data available on pressure, tank walls' defor mation or cavity evolution of the HRAM phenomenon, experi mental tests were made by the same workers and their results were presented in a previous paper [29]. The sketch of the experimental device used for impact tests is shown in Fig. 2.

The test boxes consisted of $6063 \mathrm{~T} 5$ square aluminium tubes $750 \mathrm{~mm}$ long, $150 \mathrm{~mm}$ wide and $2.5 \mathrm{~mm}$ thick. The specimens were closed with two PMMA windows $30 \mathrm{~mm}$ thick, fixed to the specimen with four steel bars; these transparent panels allow for the recording of the impact process, by means of a Photron Ultima APX RS digital high speed camera. Lighting was provided by an Arrisun 12 Plus lamphead with a $1200 \mathrm{~W}$ Hydrargyrum Medium arc Iodide (HMI) lamp. A similar setup was proposed by Nishida and Tanaka [30]. The contact points between PMMA windows and specimen were sealed with silicone in order to avoid fluid leakage [19].

Pressure data in the fluid was obtained at two different points, PTn (near the impact) and PTf (far from the impact), by means of a PCB 138A06 transducer [19]. Two holes were made on the lower wall of the specimen to place both sensors inside the water. Their position is shown in Fig. 3. A Dewetron DEWE 800 data acquisition device was used to record the signals. This system can record at a sampling rate of $1 \mu \mathrm{s}$ and synchronise the data with the video recording.
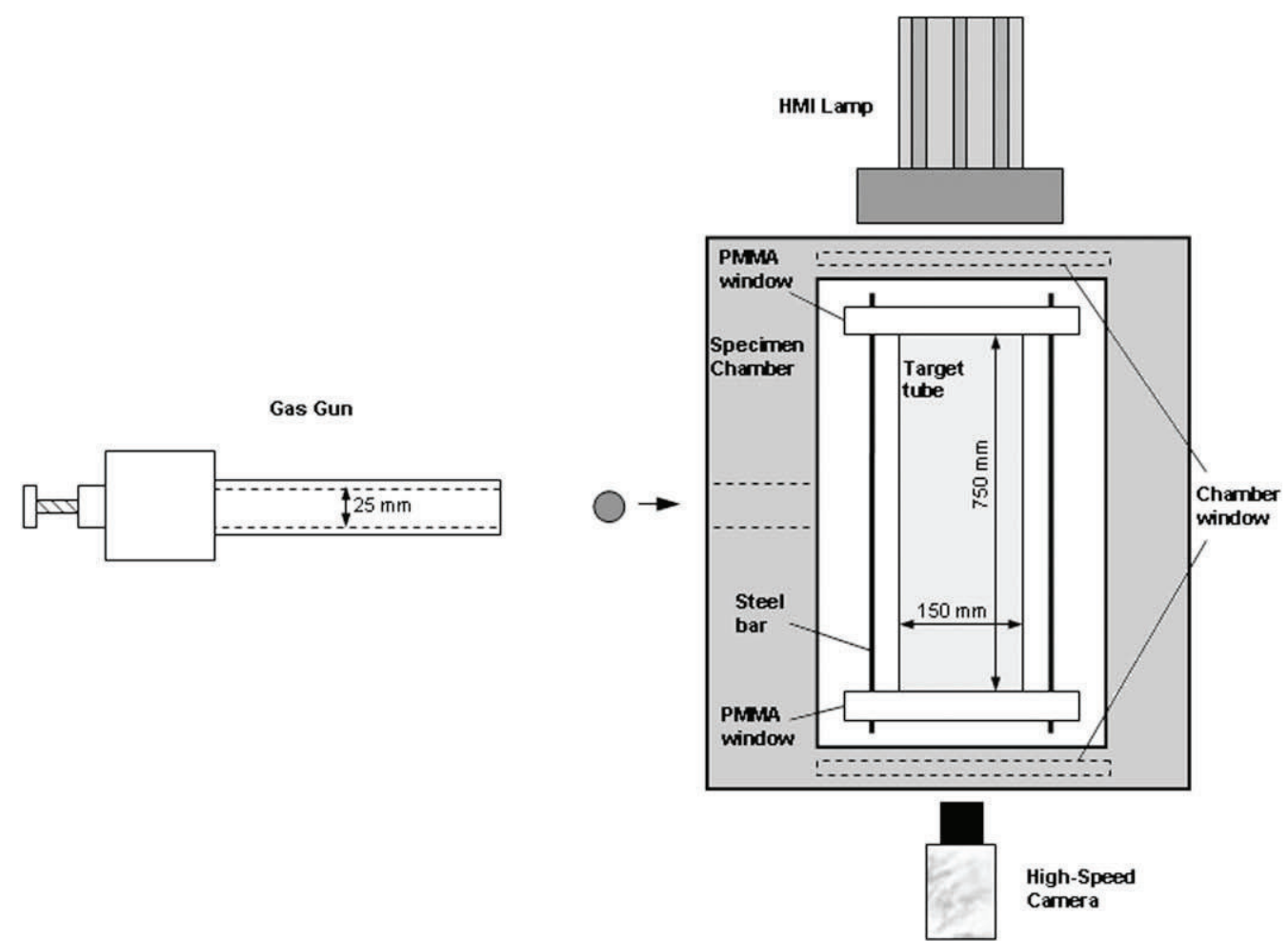

Fig. 2. Sketch of the experimental device used for the impact tests [ref nuestro articulo]. 


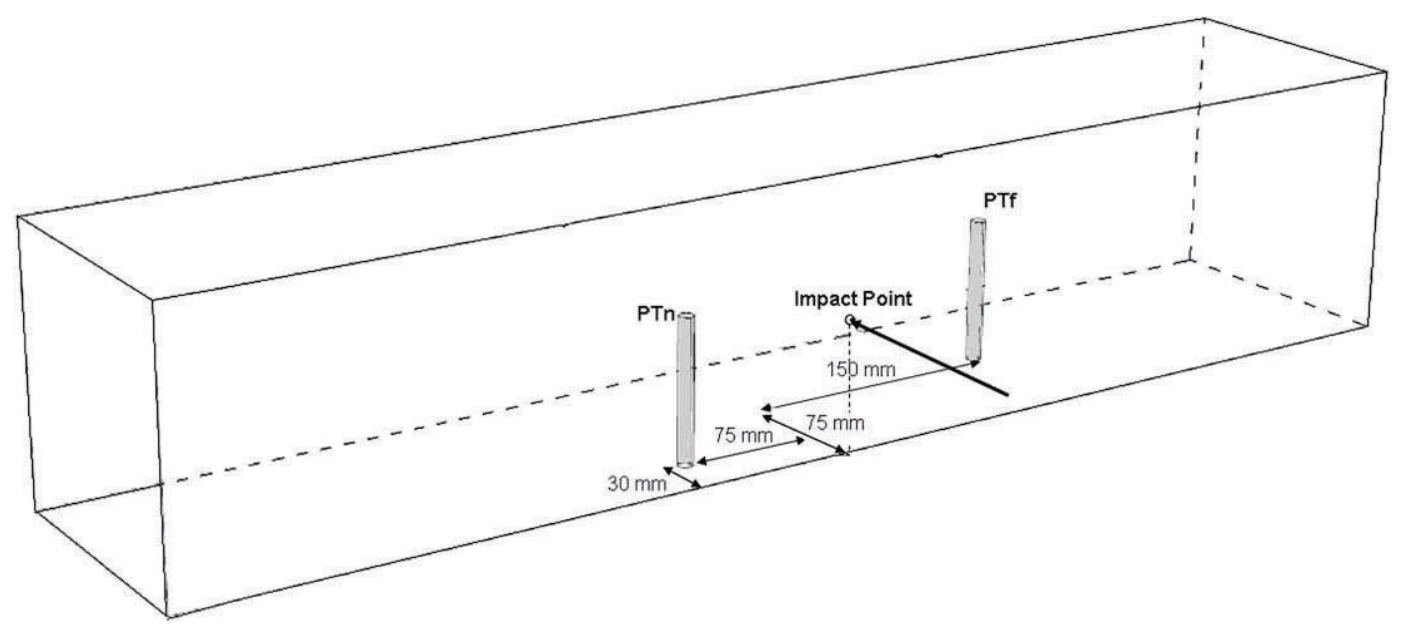

Fig. 3. Sketch of the test box instrumented.

A one stage light gas gun with a 4.71 chamber which stores gas at a maximum pressure of 300 bar was used with helium. The length of the barrel was $4.5 \mathrm{~m}$ and its calibre $25 \mathrm{~mm}$. The gun was aimed at an armoured steel specimen chamber box $1 \times 1 \times 1 \mathrm{~m}^{3}$ where the specimen was placed during the impact test. The chamber had a small circular window in the front for the projectile to pass through, and two large lateral windows to illuminate the specimen and capture the video sequence of the impact, Fig. 2.

The projectile launched against the box was a steel sphere with a diameter of $12.5 \mathrm{~mm}$ and a mass of $8 \mathrm{~g}$. Two impact velocities were performed: 600 and $900 \mathrm{~m} / \mathrm{s}$.

\section{Numerical analysis}

The numerical models were developed with the commercial finite element code LS DYNA v.971 [18]. This software is particu larly suitable for nonlinear dynamic problems, as is the case of impacts or explosions. It also allows for the employment of different techniques such as ALE or SPH to solve fluid structure problems, which makes it ideal to compare both approaches. In order to reproduce the HRAM phenomenon, the ALE technique and the SPH method were adopted to model the fluid inside the tank.

\subsection{Box and projectile FE model}

The symmetry of the problem under consideration allowed modelling only a quarter of the whole (Fig. 4, Left). Since the nature of this simulation demands a very high mesh density, such a reduction in the model size is very desirable. The box has been divided into three parts, the walls impacted by the projectile (entry and exit walls), the lateral wall and the PMMA window.

The impacted walls and the PMMA window were discretised by means of eight node solid hexahedron Lagrangian elements with reduced integration. A refined mesh, corresponding to the impact zone, and a progressively coarser mesh as the distance to the hit point grows can be observed in Fig. 4, right. The impacted walls present five elements through the thickness and an element of $1 \mathrm{~mm}$ in size in the other two directions near the impacted zone. Based on the previous simulations, the mesh size was considered appropriate to reproduce the behaviour of the solids in the impacted zone. Four noded Belytschko Tsay shell elements were used to discretise the lateral wall in order to reduce the number of elements. Finally, the mesh of the box consisted of 15902 elements.

The Johnson Cook hardening relation [31] was selected to model the aluminium of the box. There are more sophisticated hardening relations, but Johnson and Cook's is probably the most
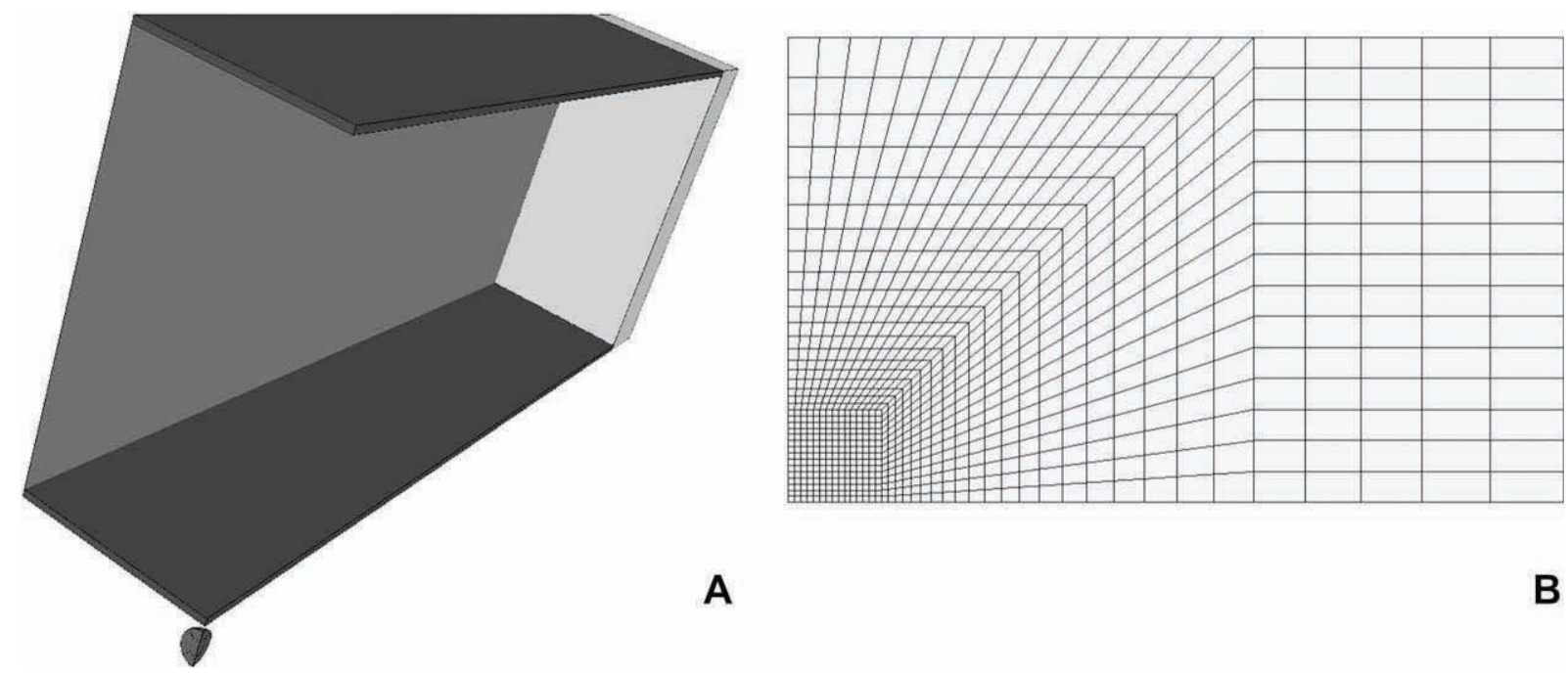

Fig. 4. Left: box model geometry used for the analysis. Right: details of the entry wall mesh. 
Table 1

Parameters used in the simulation for the solids

\begin{tabular}{llllllll}
\hline Material & $\rho\left(\mathrm{kg} / \mathrm{m}^{3}\right)$ & $E(\mathrm{GPa})$ & $\nu$ & $A(\mathrm{GPa})$ & $B(\mathrm{GPa})$ & $n$ & \\
\hline 6063-T5 & 2700 & 71 & 0.33 & 0.2 & 0.144 & 0.62 & - \\
Steel & 7830 & 207 & 0.28 & - & - & - & - \\
PMMA & 1180 & 3 & 0.35 & - & - & - & - \\
\hline
\end{tabular}

widely used among those accounting for equivalent plastic strain $\bar{\varepsilon}^{\mathrm{p}}$, equivalent plastic strain rate $\cdot \bar{\varepsilon}^{\mathrm{p}}$ and temperature effects $\theta$. Since numerous efforts have been made in the past to determine their parameters for a large number of metallic materials, it has been implemented in many FE explicit codes. The relation is stated through the following multiplicative equation:

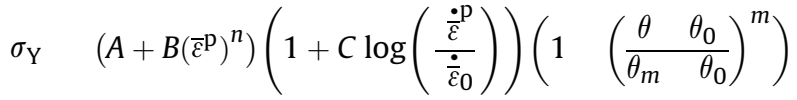

$\theta_{0}$ being the reference temperature and $\theta_{m}$ the melting tempera ture. The required parameters of the A6063 T5 were obtained from Refs. [32,33]. In order to describe the material failure in the zone around the impact point, the Johnson Cook model was used; the relation is described by the following equation:

$$
\begin{array}{ll}
\varepsilon_{\mathrm{f}} \quad & \left(D_{1}+D_{2} \exp \left(D_{3} \frac{\sigma_{\mathrm{h}}}{\sigma_{\mathrm{eff}}}\right)\right)\left(1+D_{4} \ln \frac{\dot{\bar{\varepsilon}}}{\dot{\bar{\varepsilon}}_{0}}\right) \\
& \times\left(1+D_{5}\left(\begin{array}{ll}
\theta & \theta_{0} \\
\theta_{m} & \theta_{0}
\end{array}\right)\right)
\end{array}
$$

where $\sigma_{\text {eff }}$ and $\sigma_{\mathrm{h}}$ are, respectively, the effective and the hydrostatic stresses; the failure occurs when the damage parameter $\sum \Delta \bar{\varepsilon}^{\mathrm{p}} / \varepsilon_{\mathrm{f}} \quad 1$. In this case the failure model was used without taking into account the sensibility of temperature, strain rate and triaxiality, and hence the constants $D_{2}, D_{3}, D_{4}$ and $D_{5}$ are set to zero. The fail will occur when $\bar{\varepsilon}^{\mathrm{p}}$ reaches the value of $D_{1}$.

An elastic material model was used for the PMMA window [34]. The projectile was discretised by means of eight node solid hexa hedron Lagrangian elements with reduced integration and modeled as an elastic material with the steel characteristic parameters since no plastic deformation was observed in the tests. The material properties and parameters used are shown in Table 1.

\subsection{Models for the fluid}

It is expected that the fluid inside the tank undergoes defor mations too large to consider a pure Lagrangian description as an appropriate option. For this reason, a multi material ALE formula tion or a mesh free approach (SPH) has been chosen for the treatment of the fluid. Multi material means that each element of the mesh has the ability to contain two or more materials, in this case water and air. The ALE formulation allows the motion of the mesh independently of the material flow without distortion prob lems. The SPH approach implies that the fluid is modeled by means of particles which are not fixed by connectivity constraints, there fore they are able to freely move and deform in any way repro ducing fluid behaviour.

\subsubsection{ALE model}

The fluid inside the box is discretised by means of eight node solid hexahedron elements with an ALE formulation (elform 11 in LS DYNA notation). Strictly, the fluid is discretised by means of an Eulerian mesh, but LS DYNA uses an ALE formulation in multi material problems since it considers an Eulerian part as a special ALE case where the mesh velocity is zero. In this case, the air surrounding the box was also considered, being modeled using the same elements as in the water. Modelling this air region is essential to allow the water to flow into it, deforming the walls of the structure. This is only possible if the water and air meshes share the same nodes at their interface. Four discretization densities were analyzed in order to achieve an optimal mesh density; a simplified model without the aluminium tube was used to perform this analysis. The results in terms of projectile deceleration were compared with the classical solution of the movement of a sphere inside a liquid. Finally, the fluid inside the box and the surrounding air region resulted in 61519 elements, Fig. 5. Previous tests were made with a finer mesh, but instability and leakage problems at the fluid/solid interfaces appeared. To avoid these problems, numerous iterations modifying some of the coupling parameters were made, and finally it was decided to modify the mesh size in order to match the Lagrangian one at the interfaces. This change in the mesh hel ped to control the leakage problems. In addition a second order accurate advection method has been chosen.

The water was modeled using the following viscous constitutive equation (Material Null in LS DYNA notation)

$\sigma_{i j} \quad 2 v_{\mathrm{d}} \ddot{\varepsilon}_{i j}^{\prime} \quad P \delta_{i j}$

in which $\nu_{\mathrm{d}}$ is the dynamic viscosity, $\cdot \varepsilon_{i j}^{\prime}$ the deviatoric strain rate, and $I$ the identity tensor. The pressure $P$ is calculated as a function of the compression $\mu \quad \rho / \rho_{0} \quad 1$, where $\rho$ and $\rho_{0}$ are the current and initial densities of the material, respectively, and of the internal energy per unit volume $E$, using the Mie Gruneisen Equation of State based on a cubic shock velocity particle velocity

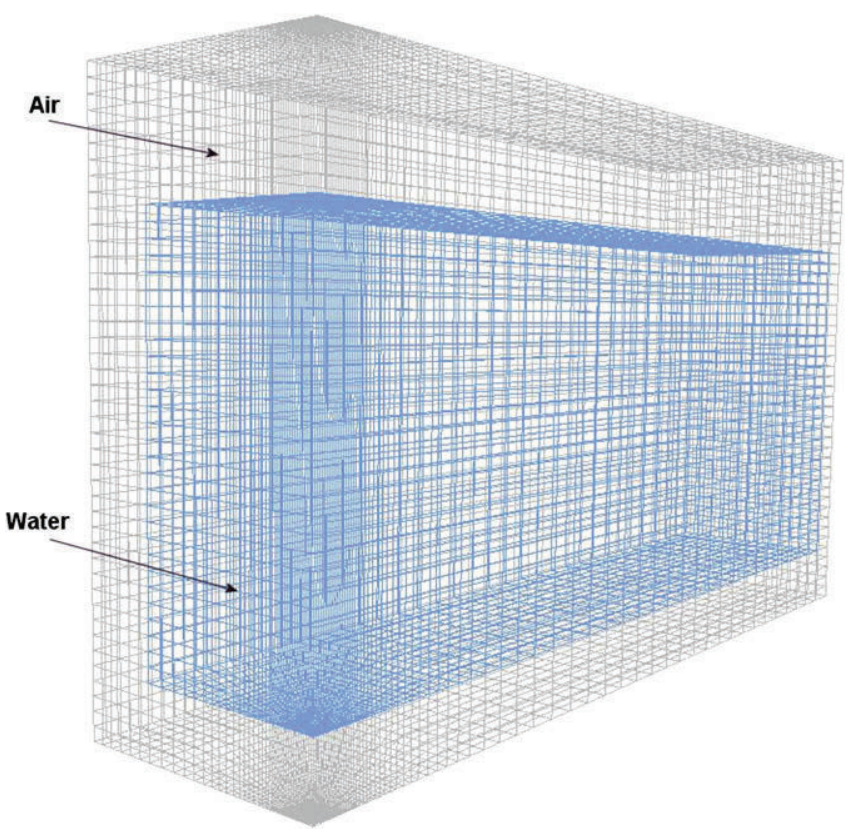

Fig. 5. Mesh of the fluids in the ALE approach. 
Table 2

Water and air parameters used in the simulation for ALE and SPH meshes

\begin{tabular}{lllllllllll}
\hline & $\rho_{0}\left(\mathrm{~kg} / \mathrm{m}^{3}\right)$ & $\nu_{\mathrm{d}}(\mathrm{Pa} \mathrm{s})$ & $C(\mathrm{~m} / \mathrm{s})$ & $S_{1}$ & $S_{2}$ & $S_{3}$ & $\gamma_{0}$ & $a$ & $C_{4}$ & $C_{5}$ \\
\hline Water & 1000 & $0.89 \times 10^{3}$ & 1448 & 1.979 & 0 & 0 & 0.11 & 3.0 & - \\
Air & 1.22 & $1.77 \times 10^{5}$ & - & - & - & - & - & - & 0.4 \\
\hline
\end{tabular}

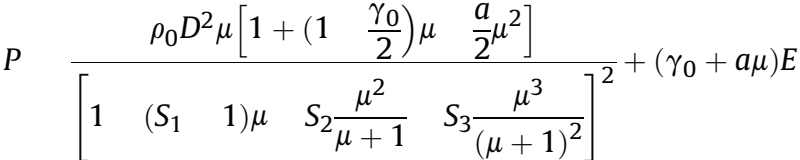

for compressed materials and

$P \quad \rho_{0} D^{2} \mu+\left(\gamma_{0}+a \mu\right) E$

for expanded materials, where $S_{1}, S_{2}$ and $S_{3}$ are coefficients of the slope of the $u_{\mathrm{s}} u_{\mathrm{p}}$ curve, $u_{\mathrm{s}}$ and $u_{\mathrm{p}}$ being the shock velocity and particle velocity, respectively. $D$ is the intercept of the $u_{\mathrm{s}} u_{\mathrm{p}}$ curve, which corresponds to the adiabatic sound speed on water. $\gamma_{0}$ is the Gruneisen gamma and $a$ is the first volume correction to $\gamma_{0}$. The required properties and constants of water were obtained from Ref. [35].

The air was modeled using the same constitutive Eq. (3), with the properties of the air, and a Linear Polynomial Equation of State [18]. The mentioned Equation of State is linear in the internal energy and polynomial in the compression, and defines the pres sure $P$ as follows:

$P \quad C_{0}+C_{1} \mu+C_{2} \mu^{2}+C_{3} \mu^{3}+\left(C_{4}+C_{5} \mu+C_{6} \mu^{2}\right) E$

The air was considered as an ideal gas by setting $C_{0} \quad C_{1}$

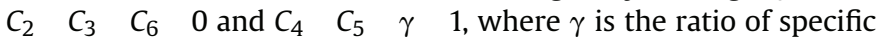
heats:

$\gamma \quad \frac{C_{\mathrm{p}}}{C_{\mathrm{V}}}$

and the pressure $P$ is given by:

$P \quad(\gamma \quad 1) \frac{\rho}{\rho_{0}} E$

The properties and parameters used in the simulation for the water and the air are shown in Table 2.

The fluid structure interaction, for both projectile/fluid and walls/fluid, is achieved by means of a penalty based ALE Lagrangian coupling algorithm implemented within LS DYNA. This allows the
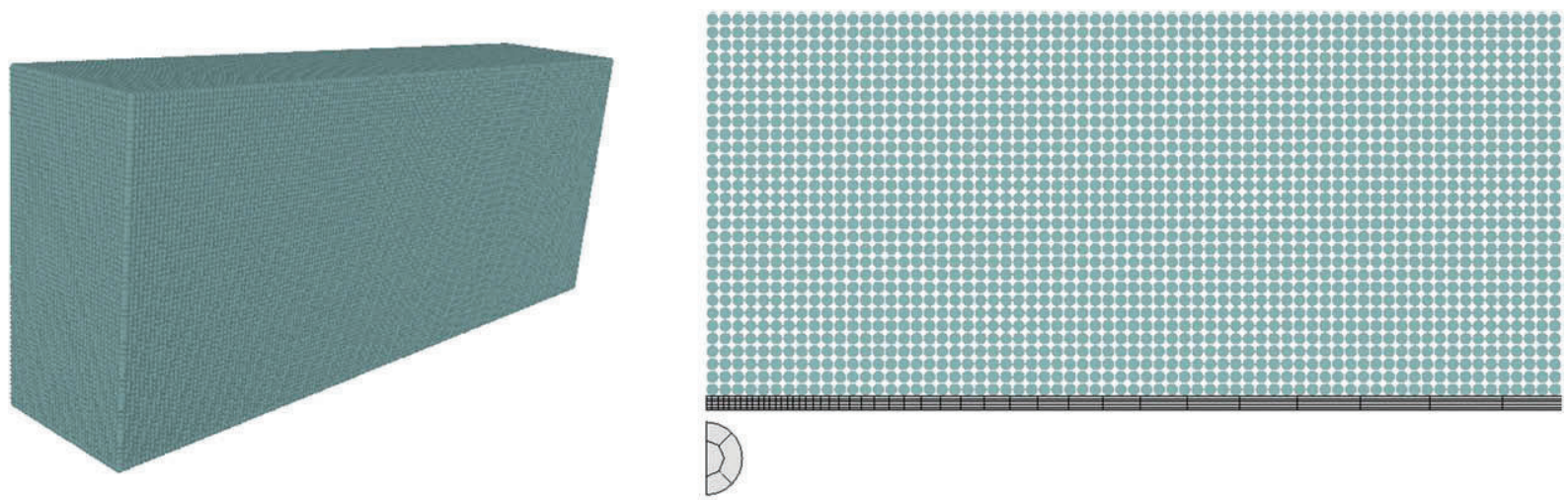

Fig. 6. Left: mesh of the water in the SPH approach. Right: details of the SPH mesh.
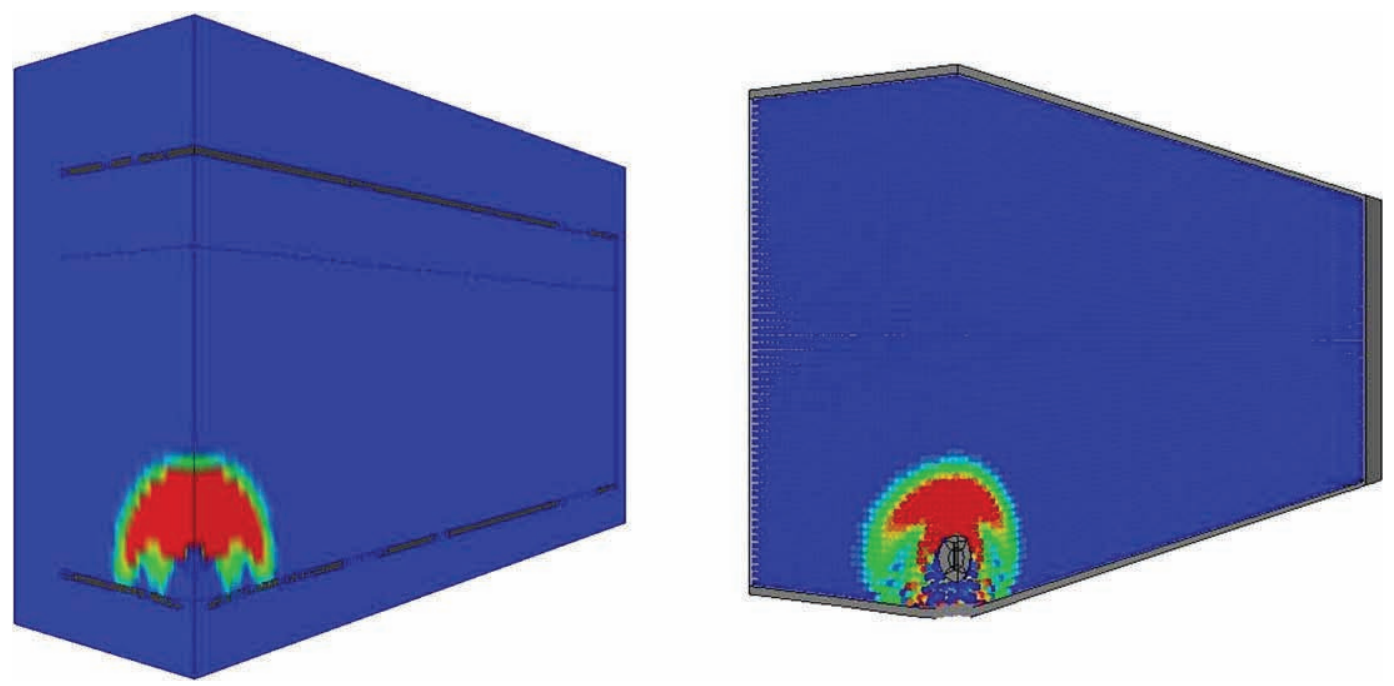

Fig. 7. Shock pressure at $t \quad 0.03$ ms. Left: ALE simulation. Right: SPH simulation. 


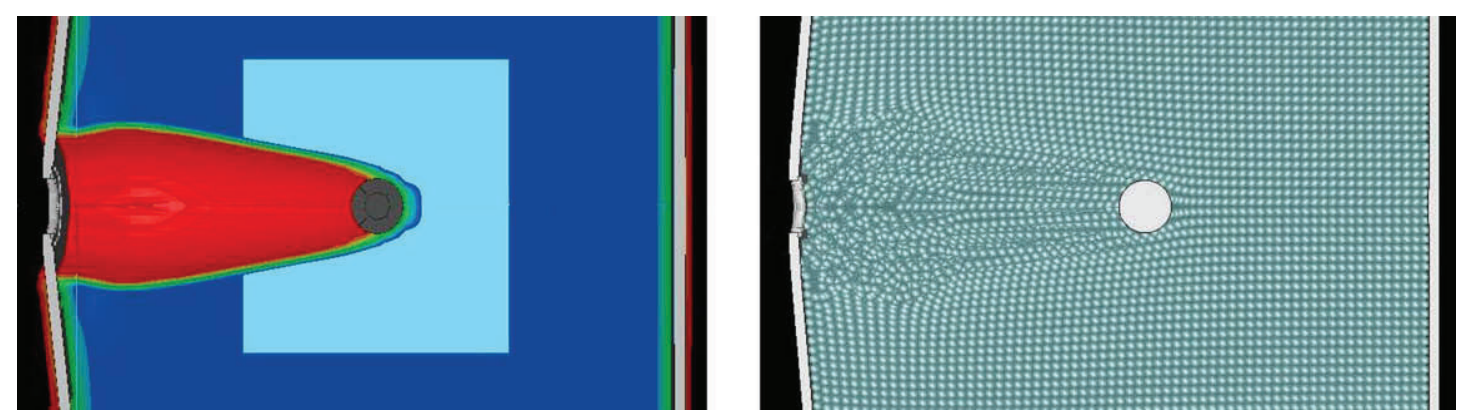

Fig. 8. Cavity formed in the drag phase at $t \quad 0.12 \mathrm{~ms}$. Left: ALE simulation. Right: SPH simulation.
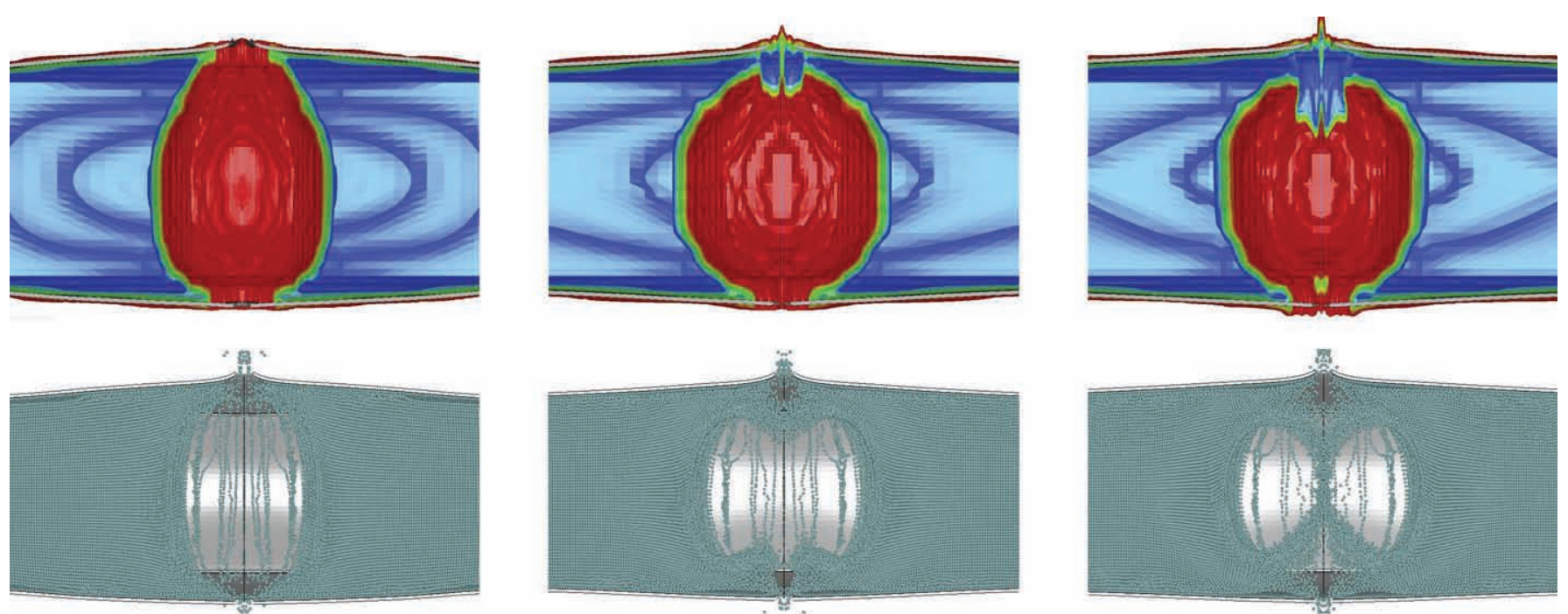

Fig. 9. Expansion and beginning of collapse of the cavity at time 1.5, 3.5 and $6.5 \mathrm{~ms}$.
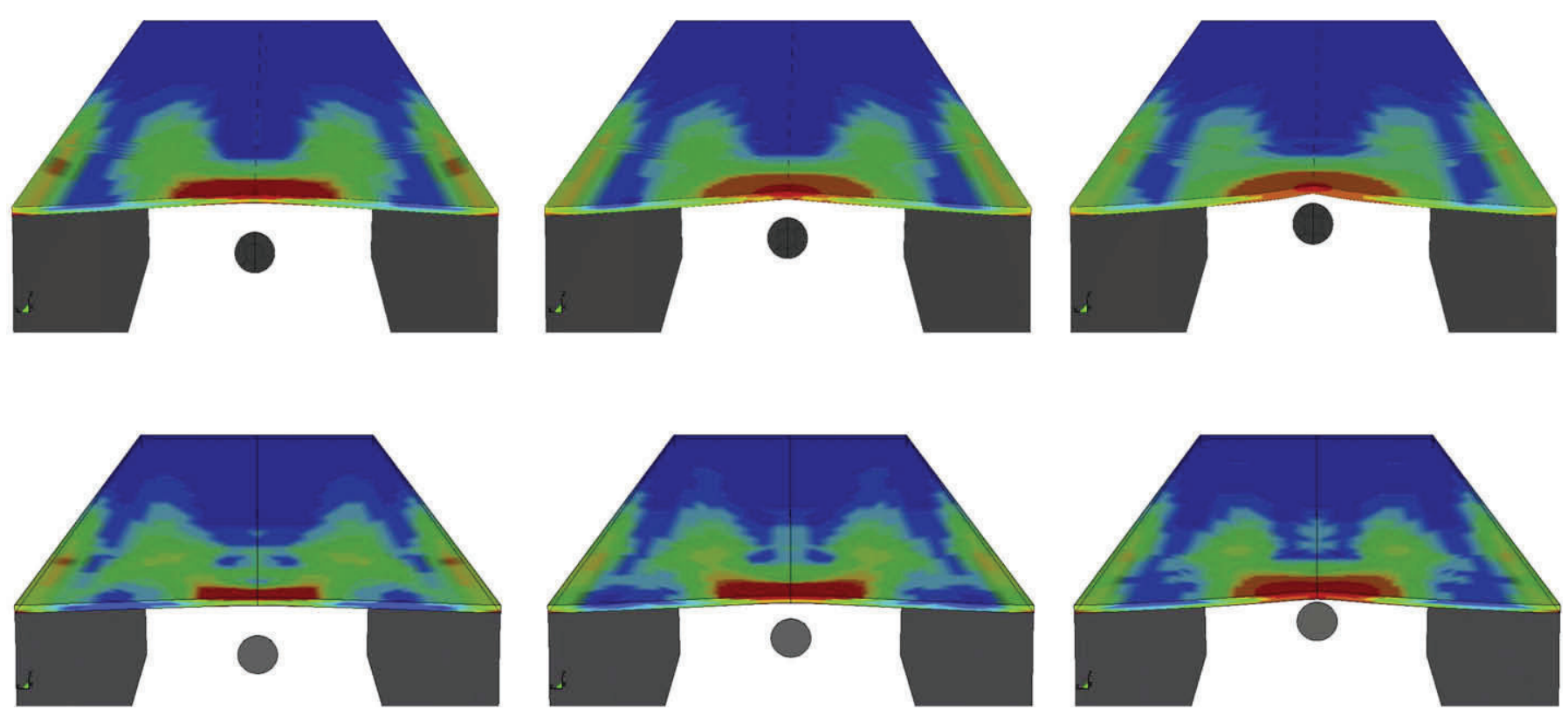

Fig. 10. Contours of effective stress in the exit wall before being impacted. Top: ALE simulation at time $0.24,0.25$ and 0.26 ms. Bottom: SPH simulation at times $0.21,0.22$ and $0.23 \mathrm{~ms}$. 

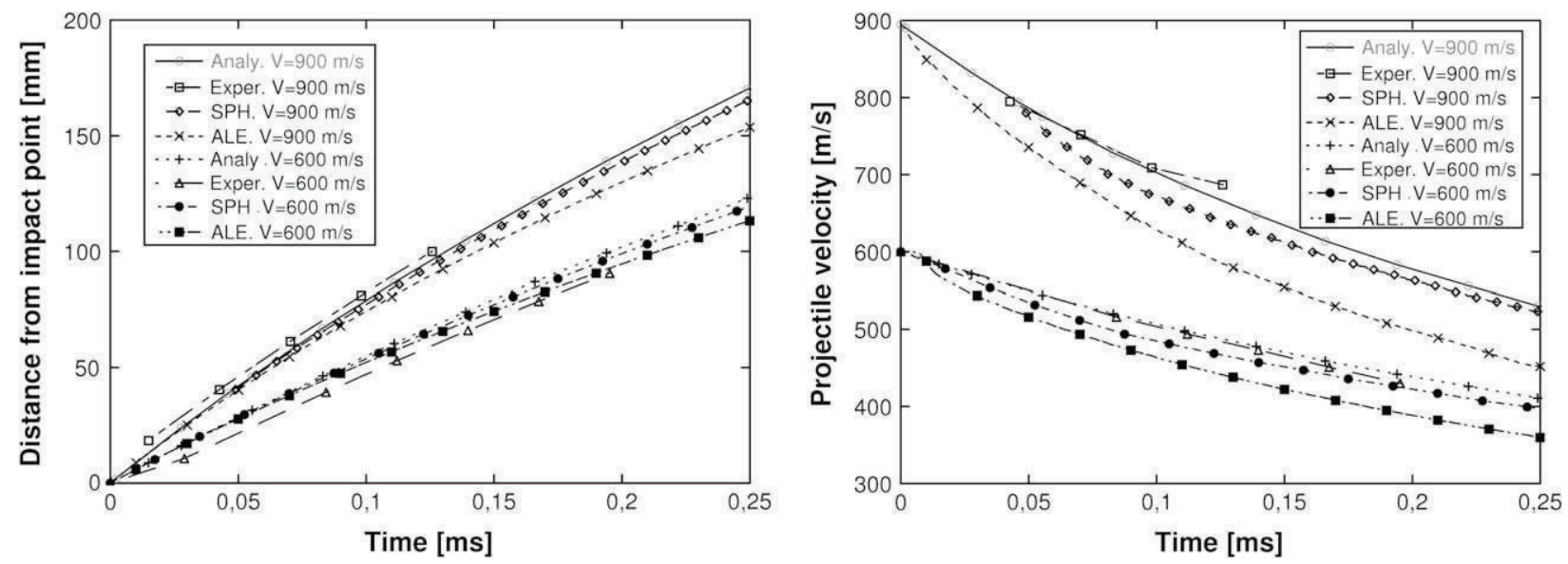

Fig. 11. Left: comparison of position of the projectile vs. time. Right: comparison of velocity decay vs. time.

fluid material to flow around the structure, but prevents its pene tration into the structure mesh applying penalty forces to the fluid and the structure. When a fluid particle penetrates the Lagrangian structure, a force of recall is applied to both the fluid particle and the structural node to make the fluid particle return to the surface of the structure avoiding penetration. The penalty forces are propor tional to the penetration depth and penalty stiffness, behaving like a spring system.

\subsubsection{SPH model}

In this case, the fluid inside the box is discretised by means of a set of particles assigned with a mass interacting among them selves without a direct connectivity. The SPH method requires a large number of particles uniformly distributed to provide reasonably accurate results. Several particle sizes were analyzed in order to achieve an optimal mesh density; as for the ALE mesh, a simplified model without the aluminium tube was used to perform this analysis. Six different particle sizes were studied and finally a diameter of $2.23 \mathrm{~mm}$ was employed, resulting in 381480 particles (Fig. 6). In the SPH method, it is not necessary to model the surrounding air since the particles can freely flow in any direction deforming the walls of the structure. The constitutive law and the equation of state used to model the water were the same as in the ALE approach (Table 2).

This time, the fluid structure coupling algorithm is different from the one applied with the ALE approach. For the interaction with the projectile, a penalty based node to surface contact interface has been used. In all the contact algorithms, the SPH particles are considered as nodes. As other authors [36] suggest, the contact interface was chosen with special care in order to
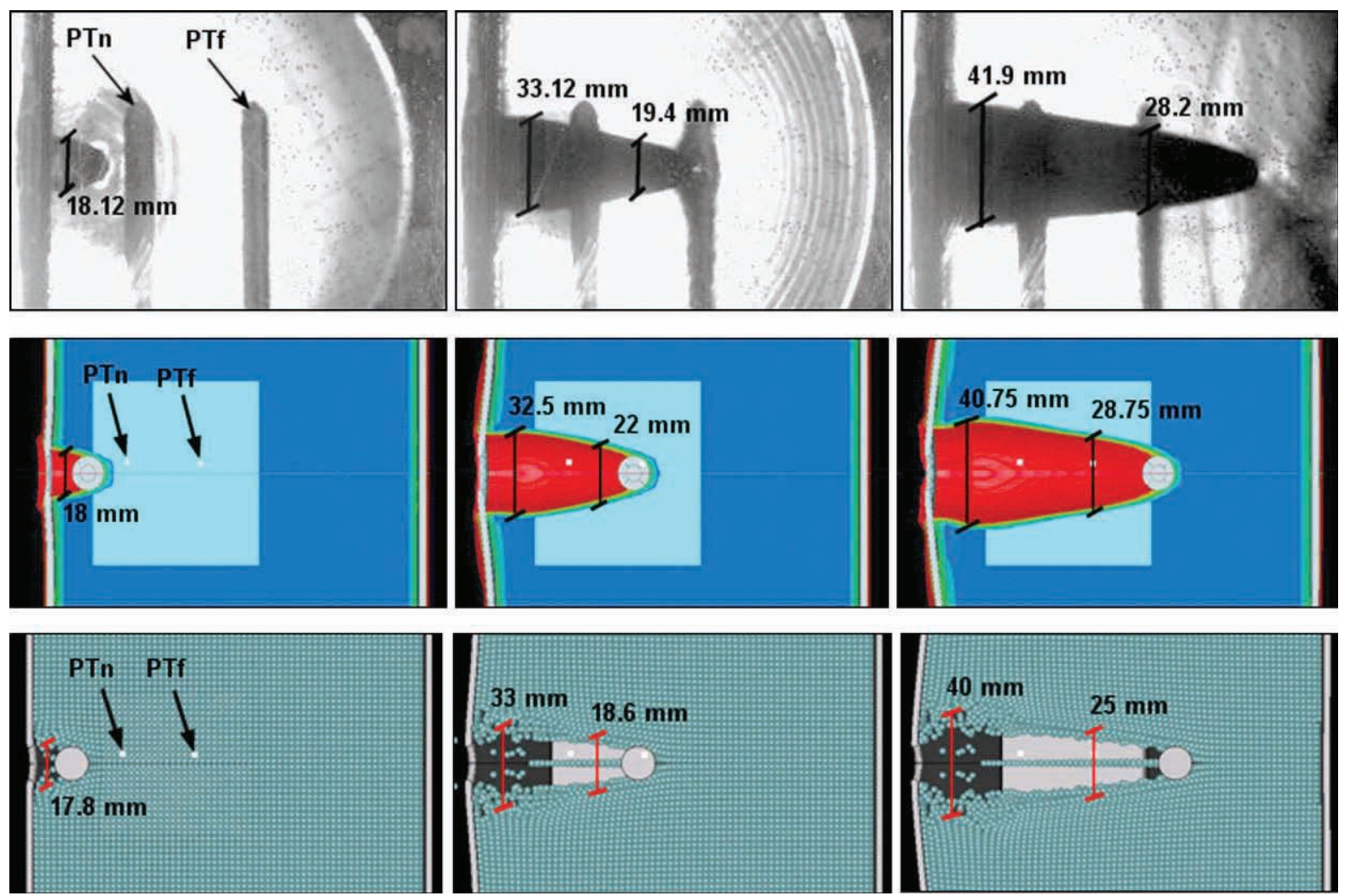

Fig. 12. Cavity evolution at time $0.028,0.084$ and $0.140 \mathrm{~ms}$ obtained from experiments (top), ALE simulation (centre) and SPH simulation (bottom). 
achieve the interaction of several SPH particles with each face of the elements of the Lagrange contacting body. This fact, linked to the necessity of a homogenous mesh to obtain accurate results, leads to a higher number of particles to discretise the water domain than in the ALE approach. The interaction of the SPH particles and the walls of the structure was made by means of a constraint interface that ties the SPH particles to the solid elements of the surrounding structure. This has been proven as the best way to transmit the fluid movement to the structure and avoid contact instabilities.

\section{Results}

In the following section, numerical results of $100 \%$ full tubes impacted at velocities of 900 and $600 \mathrm{~m} / \mathrm{s}$ are analyzed and compared with the available experimental data.

\subsection{HRAM stages}

Firstly, it has been proved that the employed approaches, ALE and $\mathrm{SPH}$, are able to qualitatively and in an appropriate way reproduce an HRAM phenomenon. The HRAM stages have been analyzed to prove that every phase is well represented in the simulations.

Shock phase. When the projectile penetrates the wall of the fluid filled structure, the impact energy is transferred to the
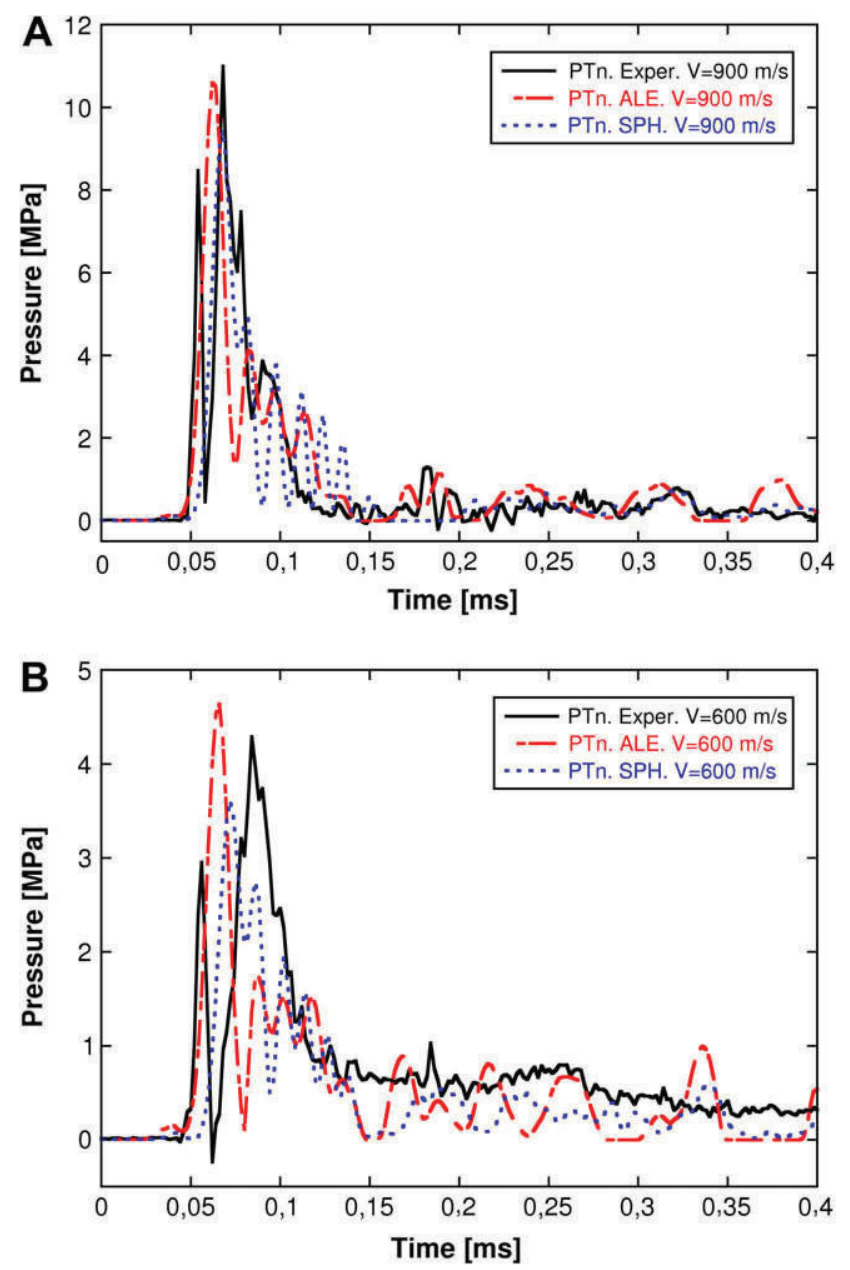

Fig. 13. Pressure time history near the impact point (PTn) in a tube $100 \%$ filled. Left: impacted at $900 \mathrm{~m} / \mathrm{s}$. Right: impacted at $600 \mathrm{~m} / \mathrm{s}$. fluid generating a high pressure hemispherical shock wave. The mentioned hemispherical shock wave is observed in both approaches, Fig. 7.

Drag phase. In the drag phase, part of projectile's kinetic energy is transformed into fluid motion. The displacement of the fluid from the projectile path generates a radial pressure field and a cavity behind it. Fig. 8 depicts the cavity formed in the ALE and SPH simulations.

Cavitation phase. The expansion and collapse (oscillations) of the cavity are known as the cavitation stage. In the employed models, the expansion of the cavity as well as the collapse is well represented, Fig. 9. It can be seen that although both approaches are capable of reproducing this phase, the SPH simulation seems to show a more realistic behaviour of this stage due to the finer and homogeneous SPH mesh.

Exit phase. The projectile exits the tank through a pre stressed wall, caused by the initial shock stage and the subsequent loading by the fluid. This is well depicted in Fig. 10, where contours of effective stress are shown in the exit wall before being penetrated by the projectile.

The contours are very similar in both approaches. The slight difference in time is due to the fact that in the ALE simulation the projectile is slowed down faster than in the SPH approach, as will be shown later, so the projectile reaches the exit wall sooner in the SPH simulation.
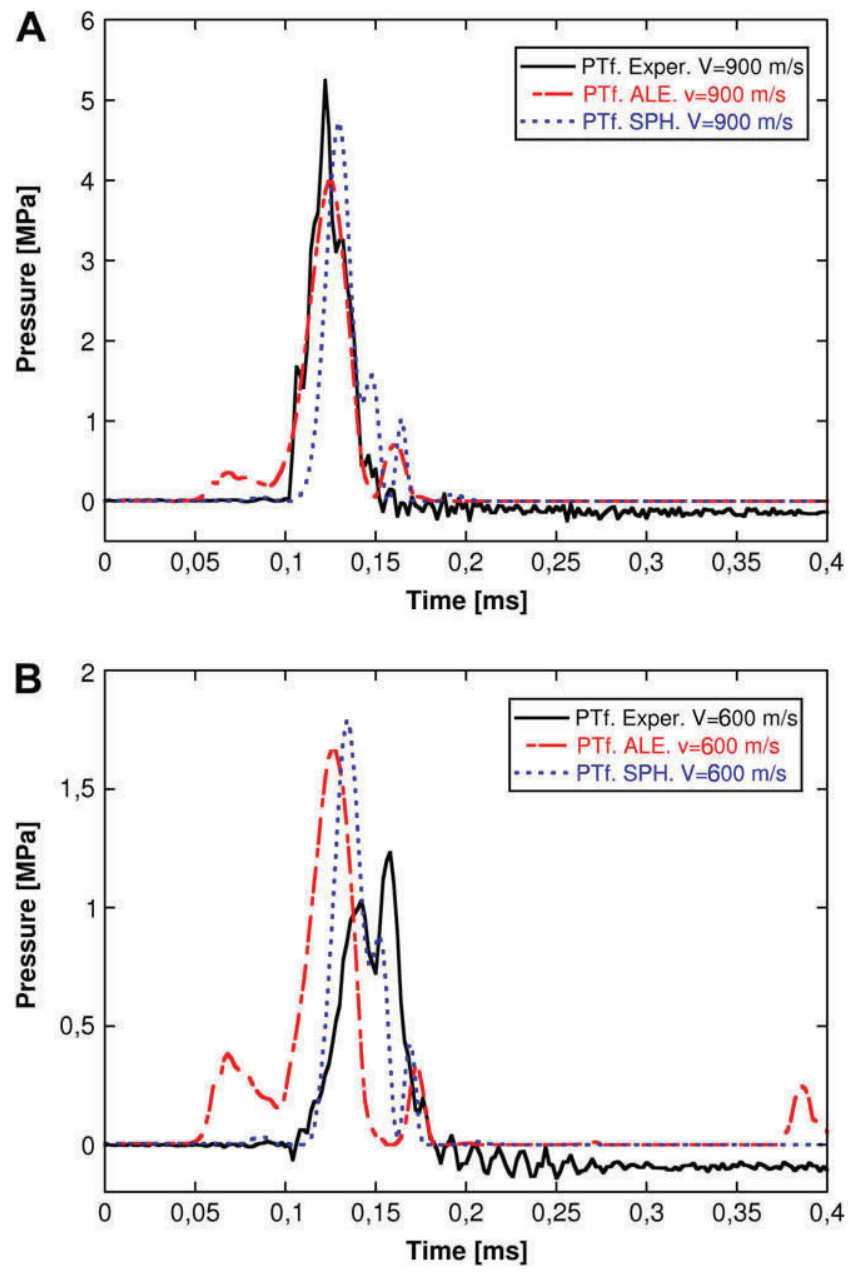

Fig. 14. Pressure time history far from the impact point (PTf) in a tube $100 \%$ filled. Left: impacted at $900 \mathrm{~m} / \mathrm{s}$. Right: impacted at $600 \mathrm{~m} / \mathrm{s}$. 


\subsection{Time history of the projectile trajectory}

By means of the digital high speed camera, it is possible to determine the velocity and the position of the projectile inside the fluid. This experimental data is compared with the numerical and analytical results [29] (Fig. 11), obtained from

$\frac{\mathrm{d} V_{\mathrm{p}}}{\mathrm{d} x_{\mathrm{p}}} \quad \frac{1}{2 m_{\mathrm{p}}} \rho_{\mathrm{w}} C_{\mathrm{d}} A_{0} V_{\mathrm{p}}$

where $m_{\mathrm{p}}$ and $V_{\mathrm{p}}$ denote the bullet mass and velocity, $\rho_{\mathrm{w}}$ is the fluid density, $A_{0}$ is the projected frontal area of the projectile and $C_{\mathrm{d}}$ is a dimensionless drag coefficient. According to the range of veloci ties considered, a value of 0.4 for $C_{d}$ was chosen [37].

The figures show a good correlation between numerical and analytical curves; the position of the projectile in the water is better predicted than the velocity. The ALE simulation predicts a lower velocity, although the difference in this last case is less than $10 \%$. This difference is due to the ALE mesh. As was mentioned before, the ALE mesh fits with the Lagrangian mesh to avoid leakage instabilities in the simulation. In preliminary tests, where a finer ALE mesh was employed (leading to instabilities), better velocity results were achieved, as also was observed by Poehlmann Martins et al. [38]. The experimental curves correlate quite well with the numerical ones showing the same trend.

\subsection{Cavity evolution}

An interesting parameter to analyse and one that has been less studied is the cavity evolution inside the fluid. Fig. 12 shows a comparison of the cavity evolution obtained both experimentally
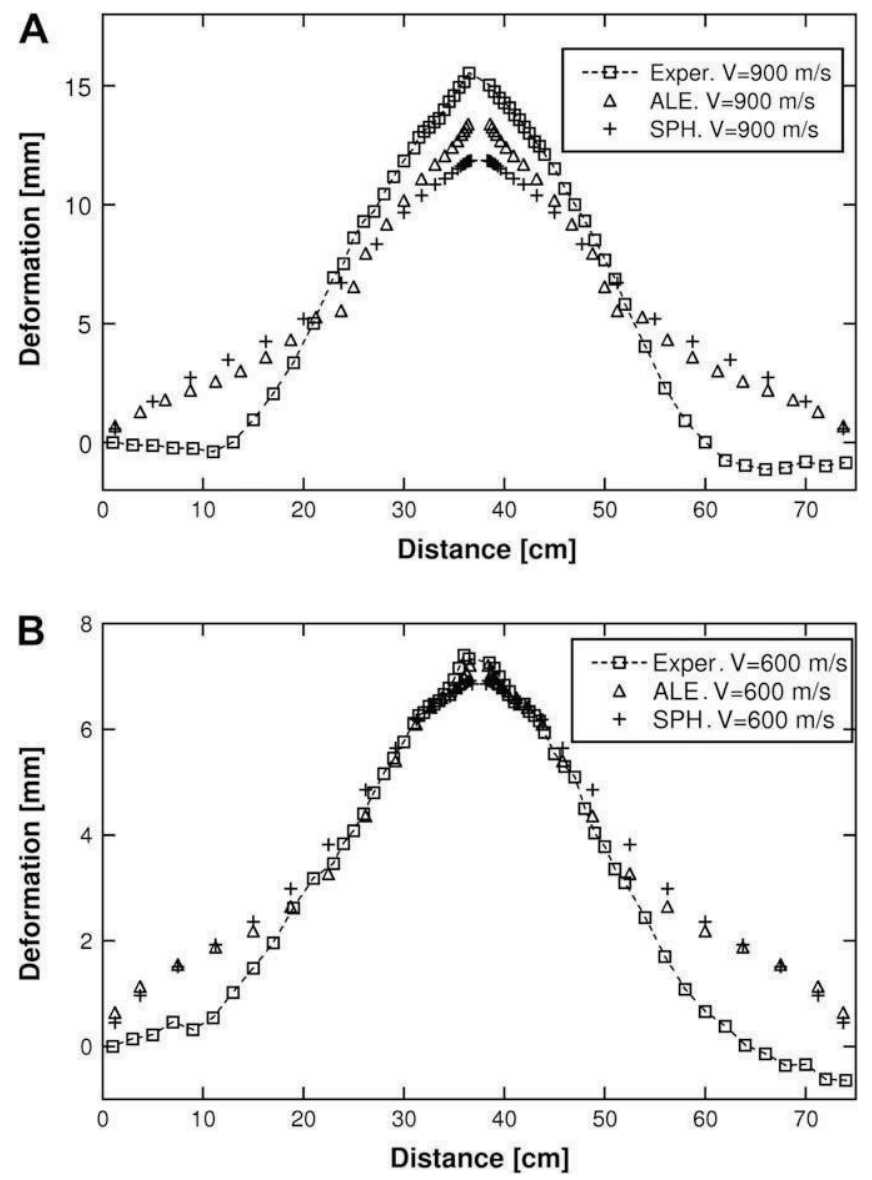

Fig. 15. Deformation of the entry wall. Left: impacted at $900 \mathrm{~m} / \mathrm{s}$. Right: impacted at $600 \mathrm{~m} / \mathrm{s}$. and numerically. It can be seen that the cavity evolution predicted by the simulations matches very well with experimental images. This is of great importance since the cavity is the main cause for the deformation of the walls.

\subsection{Pressure time history}

As mentioned before, two pressure gauges were used to record the time history of the HRAM pressure wave as it propagated through the fluid. One of the pressure gauges (PTn) was located near the impact point, at $30 \mathrm{~mm}$ from the wall and $75 \mathrm{~mm}$ from the shot line (Fig. 3), while the other (PTf) was in the middle of the tube, at $150 \mathrm{~mm}$ from the projectile trajectory (Fig. 3). The pressure time histories depicted in Figs. 13 and 14 represent the experimental and numerical curves found for pressure measurements at PTn and PTf at different velocities. The numerical curves correspond to the aver aged value of several elements around the pressure gauges' position.

It can be seen that the pressure time history changes as a func tion of the location of the pressure gauge as well as the velocity of the projectile; this is well captured by the simulations. The figures show good correlation in the pressure level predicted in the simulations and the experimental results, except in PTf for a velocity of $600 \mathrm{~m} / \mathrm{s}$ where the predicted values are overestimated by both simulations. It seems that in points near impact (PTn), the ALE approach is capable of better capturing the peak pressure value although the SPH mesh is finer in that area. Far from the point of impact (PTf), the ALE prediction is not as reliable as the SPH approach. This is probably due to the fact that the SPH mesh is finer and uniform all along the water domain, while the ALE mesh is coarser further from the point of impact. As pressures are averaged
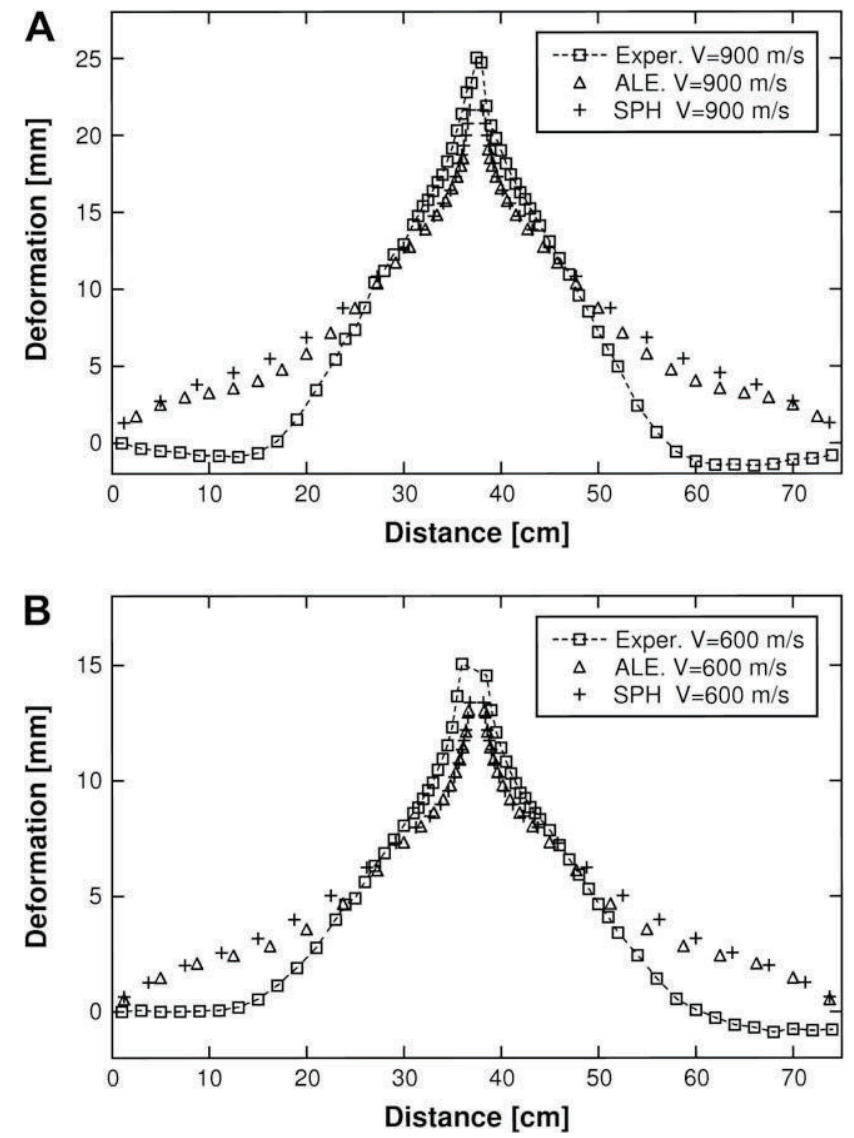

Fig. 16. Deformation of the exit wall. Left: impacted at $900 \mathrm{~m} / \mathrm{s}$. Right: impacted at $600 \mathrm{~m} / \mathrm{s}$. 
over the elements, the usage of larger elements leads to lower values of the pressure. The beginning of the pressure pulse coin cides quite well with the experimental data. The SPH simulations seem to better capture the beginning of the pulse in PTf, whereas the ALE approach predicts the beginning of the pulse a bit earlier. This could also be due to the larger size of the ALE elements.

\subsection{Deformations}

In this section, experimental deformation data in the entry and exit walls of the tubes are compared with the simulation results. Walls' deformation is interesting because of its influence on the behaviour of contiguous cells that can be part of a whole fuel tank system. Figs. 15 and 16 depict the deformation of the entry and exit walls, respectively, all along the tube at different impact velocities. The time in the simulations was long enough to assure the stabi lization of the displacements in the walls.

The figures depict how the maximum deformation is quite well predicted in the simulations. It can be observed that the shape in the nearest area to impact (about $15 \mathrm{~cm}$ at both sides of the impact point) is well represented in the simulations. The deformation at points far from the impact point is overestimated in the simulations.

\subsection{CPU time}

The CPU time required for the different approaches has been considered. The simulations were carried out using a workstation with four dual core processors (running at $2.5 \mathrm{GHz}$ ) and $16 \mathrm{~GB}$ of RAM memory. An 8 ms real time simulation took two days with the ALE approach, while the same simulation with SPH lasted one week.

The finer meshing of the SPH approach, necessary for effective simulation, requires very long CPU times, however, the results are not more accurate than the ALE ones, as has been shown. Thus, it seems that the ALE approach is more appropriate to simulate this kind of problem where a large physical domain needs to be modeled.

\section{Conclusions}

The capability of different simulation approaches in simulating the hydrodynamic ram phenomenon was studied in this work. Two different discretization techniques to solve the fluid phase were analyzed: SPH and ALE. Results of both approaches were compared with the experimental results in water filled aluminium square tubes, impacted at different velocities. The main conclusions of this work could be summarized as follows:

Both approaches ALE and SPH are capable of faithfully repro ducing the four well known stages of the hydrodynamic ram: shock, drag, cavitation and exit phases from a qualitative and quantitative perspective. The cavity evolution, main cause of the tank final deformation, is accurately reproduced as compared with the images taken from the experimental tests. In order to predict the evolution of the projectile, both types of meshes accurately describe its deceleration. Nevertheless, small differences are found in the ALE mesh due to discrep ancies in the element size compared with the SPH particles. The pressure time history at different points in the fluid, a variable of utmost importance in this kind of problems, is well predicted. Some differences are found, mainly because the shock wave propagation requires a very fine mesh to describe the pressure jump on it.
When the element size used in both SPH and ALE meshes is similar, the pressure field is better described by the ALE approach.

The final deformation of the aluminium square tube at the inlet and outlet walls is reproduced with good correlation when compared with the experimental tests. This variable is of great importance in analyzing the possible interaction with adjacent cells of the fuel tank.

The SPH mesh requires a much higher computation effort. This fact, added to the need of a constant size of the particles along the fluid, results in a better ratio accuracy by computation time with the ALE mesh.

In the case of problems in which the analyzed domain is rela tively small, the use of SPH meshes could be an appropriate choice; differences in computation efforts between ALE and SPH diminish with the number of elements (or particles).

\section{Acknowledgements}

This research was done with the financial support of the Spanish Ministry of Education under Project reference DPI2005 06769 and of the Region of Madrid and University Carlos III of Madrid under Project reference CCG07 UC3M/DPI 3395.

\section{References}

[1] Santini P, Palmieri D, Marchetti M. Numerical simulation of fluid-structure interaction in aircraft fuel tanks subjected to hydrodynamic ram penetration. In: 21st ICAS Congress, Melbourne, Australia: ICAS; 1998.

[2] Ball RE. A computer program for the geometrically nonlinear static and dynamic analysis of arbitrarily loaded shells of revolution, theory and user's manual. NASA: CR-1987; April 1972.

[3] Ball RE. Aircraft fuel tank vulnerability to hydraulic ram: modification of the northrop finite element computer code BR-1 to include fluid-structure interaction: theory and user's manual for BR-1HR, NPS-57B p74071; July 1974.

[4] Ball RE. Structural response of fluid-containing-tanks to penetrating projectiles (hydraulic ram): a comparison of experimental and analytical results. Technical report, NPS-57B p76051. Monterrey, California: Naval Post Graduate School; 1976.

[5] Fry PF, Newman RK, Bless SJ. Measurements of hydrodynamic ram-driven panel displacements and comparison with numerical predictions by BR1A(HR) and Afton codes. In: Proceedings of the hydrodynamic ram seminar; May 1977. p. 503. Technical report AFFDL-TR-77-32, JTCG/AS-77-D-002.

[6] Bless SJ, Fry PF, Barber JP. Hydrodynamic ram driven pressure fields and panel displacements for high velocity spherical fragments. In: Proceedings of the hydrodynamic ram seminar; May 1977. p. 37. Technical report AFFDL-TR-7732, JTCG/AS-77-D-002.

[7] Lundstrom EA. Fluid/structure interaction in hydraulic ram. In: Proceedings of the hydrodynamic ram seminar; May 1977. p. 223-30. Technical report AFFDLTR-77-32, JTCG/AS-77-D-002.

[8] Lundstrom EA. Structural response of flat panels to hydraulic ram pressure loading. NWC TP 6770. Naval Weapons Center; 1988. p. 12-15.

[9] Wierzbicki T, Moussa NA. Deformation and rupture of an aircraft fuel tank under hydraulic ram pressure loading. In: Proceedings of the 63rd shock and vibration symposium, vol. 2. Las Cruces, New Mexico; 1992.

[10] Herlin WM, Avery JG. Hydraulic ram structural response computer program (HRSR), Boeing Co., Prepared under Contract N60530-80-C-0242 for Naval Weapons Center, China Lake, California; 1981.

[11] Freitas CJ, Anderson Jr CE, Walker JD, Littlefield DL. Hydrodynamic ram: a benchmark suite, structures under extreme loading conditions, PVP, vol. 325 New York: ASME; 1996. p. 63-74.

[12] Kimsey KD. Numerical simulation of hydrodynamic ram. Technical report, ARBRL-TR-02217, US Army Ballistic Research Laboratory; February 1980.

[13] Anghileri M, Castelleti L-ML, Tirelli M. Fluid-structure interaction of water filled tanks during the impact with the ground. International Journal of Impact Engineering 2005;31(3):235-54.

[14] Seddon CM, Moodie K, Thyer AM, Moatamedi M. Preliminary analysis of fuel tank impact. International Journal of Crashworthiness 2004;9(3):237-44.

[15] Jarzab WW, Chwalinski R, Pfrang WE, Tokar G. Fluid-structure interaction effects in tank structures due to sloshing and hydrodynamic ram coupled Lagrangian-Eulerian simulations. In: Proceedings of the international conference: Spacecraft structures and mechanical testing, Noordwijk, The Netherlands: European Space Agency (ESA); 1988.

[16] Souli M, Olovsson L, Do I. ALE and fluid-structure interaction capabilities in LS-DYNA. In: Seventh international LS-DYNA users conference, Dearborn, Michigan; May 19-21, 2002.

[17] Fasanella EL, Boinott RL, Kellas S. Test and analysis correlation of high speed impacts of ice cylinders. In: Wayne L. Mindle, editor. Ninth international LSDYNA users conference, Dearborn, Michigan; June 4-6, 2006. 
[18] LS-DYNA keyword user's manual: nonlinear dynamic analysis of structures. Version 971, vols 1 and 2. Livermore Software Technology Corporation; May 2007.

[19] Sparks CE, Hinrichsen RL, Friedmann D. Comparison and validation of smooth particle hydrodynamic (SPH) and coupled Euler Lagrange (CEL) techniques for modelling hydrodynamic ram. In: 46th AIAA/ASME/ASCE/AHS/ASC structures, structural dynamics and materials conference, Austin, Texas; April 2005. p. 18-21.

[20] Lucy LB. A numerical approach to the testing of the fission hypothesis. The Astronomical Journal 1977;82(12):1013-24.

[21] Gingold RA, Monaghan JJ. Smoothed particle hydrodynamics: theory and application to non-spherical stars. Monthly Notices of the Royal Astronomical Society 1977;181:375-89.

[22] Vignjevic R, De Vuyst T, Campbell J, Libersky L. Modelling of hydrodynamic ram using smoothed particle hydrodynamics. In: Proceedings of the fifth international conference on dynamics and control of systems and structures in space, Cambridge, UK: Cranfield University Press; 2002.

[23] Pentecote N, Kohlgrueber D. Crash on water: a highly multi-physics problem. In: EUROPAM2004, 14th European conference and exhibition on digital simulation for virtual engineering, Paris, Frankreich; October 11-13, 2004.

[24] Chen JK, Allahdadi FA, Carney TC. High-velocity impact of graphite/epoxy composite laminates. Composites Science and Technology 1997;57:1369-79.

[25] Chen JK, Medina DF. The effects of projectile shape on laminated composite perforation. Composites Science and Technology 1998;58:1629-39.

[26] Medina DF, Chen JK. Three-dimensional simulations of impact induced damage in composite structures using the parallelized SPH method. Composites Part A: Applied Science and Manufacturing 2000;31:853-60.

[27] Knight NF, Jaunky N, Lawson RE, Ambur DR. Penetration simulation for uncontained engine debris impact on fuselage-like panels using LS-DYNA Finite Elements in Analysis and Design 2000;36:99-133.

[28] Shintate K, Sekine H. Numerical simulation of hypervelocity impacts of a projectile on laminated composite plate targets by means of improved SPH method. Composites Part A: Applied Science and Manufacturing 2004;35: 683-92.
[29] Varas D, López-Puente J, Zaera R. Experimental analysis of fluid filled aluminium tubes subjected to high velocity impact. International Journal of Impact Engineering 2008;. doi:10.1016/j.jimpeng.2008.04.006.

[30] Nishida M, Tanaka K. Experimental study of perforation and cracking of waterfilled aluminum tubes impacted by steel spheres. International Journal of Impact Engineering 2006;32:2000-16.

[31] Johnson GR, Cook WH. A constitutive model and data for metals subjected to large strains, high strain rates, and temperatures. In: Proceedings of seventh international symposium, The Hague, The Netherlands; 1983. p. 1-7.

[32] Karagiozova D, Jones N. Dynamic buckling of elastic-plastic square tubes under axial impact II: structural response. International Journal of Impact Engineering 2004;30:167-92.

[33] Yang CC. Material properties of square tube. In: The ninth fundamental scientific conference of Chinese Armed Forces Academy, Taiwan, ROC, vol. 1, ppB1 11; 2002.

[34] Vesenjak M, Matthaei M, Mullerschon H, Ren Z. Fluid models in LS-DYNA and their interaction with a structure in dynamic simulations. In: Proceedings of PVP2005. ASME Pressure Vessels and Piping Division conference, Denver; 2005. PVP2005-71557/1-5, [COBISS.SI-ID 9763862].

[35] Boyd R, Royles R, El-Deeb KMM. Simulation and validation of UNDEX phenomena relating to axisymmetric structures. In: Sixth international LSDYNA users conference simulation 2000, Dearborn, Michigan; April 9-11, 2000.

[36] Schwer LE, Preliminary assessment of non-Lagrangian methods for penetration simulation. In: Eighth international LS-DYNA users conference, Dearborn, Michigan; May 2-4, 2004.

[37] Gerhart PM, Gross RJ, Hochstein JI. Fundamentals of fluid mechanics. AddisonWesley Publishing Co.; 1992 [Chapter 8].

[38] Poehlmann-Martins F, Gabrys J, Souli M. Hydrodynamic ram analysis of non-exploding projectile impacting water. In: 2005 ASME Pressure Vessels and Piping Division conference, Denver, Colorado, July 17-21, 2005, PVP2005-71658/1-7. 